\title{
Oxidative shielding and the cost of reproduction
}

\author{
Jonathan D. Blount ${ }^{1, *}$, Emma I. K. Vitikainen ${ }^{1}$, Iain Stott ${ }^{2}$ and Michael A. Cant ${ }^{1}$ \\ ${ }^{1}$ Centre for Ecology \& Conservation, College of Life \& Environmental Sciences, University of \\ Exeter, Penryn Campus, Cornwall TR10 9FE, UK \\ ${ }^{2}$ Environment \& Sustainability Institute, University of Exeter, Penryn Campus, Cornwall TR10 \\ 9FE, $U K$
}

*Author for correspondence (Tel.: +44 (0)1326 371877; E-mail: j.d.blount@exeter.ac.uk).

\begin{abstract}
Life-history theory assumes that reproduction and lifespan are constrained by trade-offs which prevent their simultaneous increase. Recently, there has been considerable interest in the possibility that this cost of reproduction is mediated by oxidative stress. However, empirical tests of this theory have yielded equivocal support. We carried out a meta-analysis to examine associations between reproduction and oxidative damage across markers and tissues. We show that oxidative damage is positively associated with reproductive effort across females of various species. Yet paradoxically, categorical comparisons of breeders versus non-breeders reveal that transition to the reproductive state is associated with a step-change reduction in oxidative damage in certain tissues and markers. Developing offspring may be particularly sensitive to harm caused by oxidative damage in mothers. Therefore, such reductions could potentially function to shield reproducing mothers, gametes and developing offspring from oxidative insults
\end{abstract}


that inevitably increase as a consequence of reproductive effort. According to this perspective, we hypothesise that the cost of reproduction is mediated by dual impacts of maternally-derived oxidative damage on mothers and offspring, and that mothers may be selected to diminish such damage. Such oxidative shielding may explain why many existing studies have concluded that reproduction has little or no oxidative cost. Future advance in life-history theory therefore needs to take account of potential transgenerational impacts of the mechanisms underlying life-history trade-offs.

Key words: ageing, fetal programming, oxidative shielding hypothesis, oxidative stress, lifehistory trade-off, reactive oxygen species.

\section{CONTENTS}

I. Introduction

II. Aims

III. Materials and methods

(1) Literature search and criteria for inclusion in the meta-analysis

(2) Data extraction and calculation of effect sizes

(3) Procedures for meta-analysis

(4) Evaluating potential sources of bias in the meta-analysis

IV. Results

(1) Reproductive effort and oxidative damage

(2) Reproductive state and oxidative damage

V. Discussion 
(1) Why is oxidative damage reduced in reproducing females?

(a) Oxidative shielding in eutherian mammals

(b) Oxidative shielding in oviparous animals

(2) Consistency and inconsistency amongst markers of oxidative damage

(3) How is oxidative damage reduced in reproducing females?

(4) How costly is the diminishment of oxidative damage?

\section{Conclusions}

VII. Acknowledgements

VIII. References

IX. Supporting information

\section{INTRODUCTION}

The cost of reproduction is a cornerstone of life-history theory. High levels of investment in current reproduction can decrease survival to the next reproduction, or reduce future reproductive success (Stearns, 1992; Williams, 1966). While such trade-offs have been extensively documented in animals, the underlying mechanisms remain unclear (Isaksson, Sheldon \& Uller, 2011; Metcalfe \& Monaghan, 2013; Selman et al., 2012; Speakman \& Garratt, 2014). Theoretically, reproduction is costly because it entails increased levels of oxidative damage (Costantini, 2008; Dowling \& Simmons, 2009; Monaghan, Metcalfe \& Torres, 2009). Reproduction incurs elevated metabolic rate, and in turn, this is predicted to result in increased generation of reactive oxygen species (ROS) which are mainly a byproduct of ATP synthesis in mitochondria. ROS are highly unstable and can cause oxidative damage to DNA, lipids and proteins. A state of oxidative stress can arise if ROS production overwhelms the body's antioxidant defences or repair mechanisms, resulting in increased levels of oxidative damage, 
and loss of cell homeostasis and function. Oxidative damage is thought to be an important mechanism underlying the gradual deterioration of bodily function that defines ageing (Halliwell \& Gutteridge, 1999), and therefore could explain the cost of reproduction (Dowling \& Simmons, 2009; Metcalfe \& Alonso-Alvarez, 2010; Metcalfe \& Monaghan, 2013; Monaghan et al., 2009; Selman et al., 2012; Speakman \& Garratt, 2014).

Empirical tests of this theory have so far yielded equivocal support, which recent reviews have attributed to limitations in study design (Isaksson et al., 2011; Metcalfe \& Monaghan, 2013; Selman et al., 2012; Speakman \& Garratt, 2014; Stier et al., 2012). For example, many early studies failed to measure markers of oxidative damage, instead reporting only measures of antioxidant protection in order to infer an individual's oxidative state. It is now generally agreed that this approach is insufficient, because levels of antioxidants may both increase and decrease during oxidative stress; markers of oxidative damage must be measured in order to determine oxidative stress (Selman et al., 2012). Several other explanations for the inability to detect an oxidative cost of reproduction have been proposed, including a failure to measure an appropriate range of tissues or markers of oxidative damage, a failure to study animals under naturalistic levels of resource limitation, or in the wild, a failure to take into account variation in the timing of measurements, and the possibility that parents may pre-emptively increase their antioxidant defences to avoid somatic damage (Garratt et al., 2013, 2011; Metcalfe \& Monaghan, 2013; Oldakowski et al., 2012; Selman et al., 2012; Stier et al., 2012). In addition, it has recently been suggested that an important limitation of existing studies is a failure to experimentally manipulate reproductive effort, leaving open the possibility that individuals may modulate current reproductive effort to maximise their lifetime reproductive success (Metcalfe \& Monaghan, 2013). Such modulation is likely to vary according to an individual's quality or 
access to resources and could give rise to positive correlations between life-history traits, and thus obscure the oxidative cost of reproduction (Metcalfe \& Monaghan, 2013). Finally, it has been suggested that conceptual models of an oxidative cost of reproduction are too simplistic, not least because ROS production is not directly proportional to metabolic rate and in some circumstances can decrease as metabolic rate increases (Selman et al., 2012; Speakman \& Garratt, 2014).

As a result of these concerns, there is a growing consensus that we need additional, carefully designed experiments that may conclusively test whether reproduction incurs increased oxidative damage (Isaksson et al., 2011; Metcalfe \& Monaghan, 2013; Selman et al., 2012; Speakman \& Garratt, 2014; Stier et al., 2012). However, at the same time, a handful of studies have recently emerged reporting the opposite pattern to that predicted: a significant decrease in oxidative damage in breeders, at least in some tissues and markers (Garratt et al., 2012, 2013, 2011; Oldakowski et al., 2012; Xu et al., 2014; Yang et al., 2013), while paradoxically showing that animals expending greater reproductive effort have raised levels of oxidative damage (Garratt et al., 2011; Speakman \& Garratt, 2014; Stier et al., 2012; Xu et al., 2014; Yang et al., 2013). Despite any failings in the design of existing studies, why oxidative damage may become both decreased and increased in reproducing animals requires explanation.

\section{AIMS}

Faced with accumulating data from different sources that report conflicting or inconsistent results, meta-analysis is a powerful tool for synthesising research evidence across independent studies (Koricheva, Gurevitch \& Mengersen, 2014). We carried out a meta-analysis, with the aim of examining whether there are consistent associations between reproduction and oxidative 
damage. Our analysis was restricted to females of homeothermic species, for which the vast majority of data are available. In line with existing theoretical paradigms, we hypothesised that individuals expending greater reproductive effort have higher levels of oxidative damage, and reproducing individuals have higher levels of oxidative damage compared with non-reproducing individuals (reviewed by Dowling \& Simmons, 2009; Metcalfe \& Alonso-Alvarez, 2010; Metcalfe \& Monaghan, 2013; Monaghan et al., 2009; Selman et al., 2012; Speakman \& Garratt, 2014). Based on the results of this analysis we propose a new hypothesis to explain why levels of oxidative damage are often decreased in breeders, while positive correlations between reproductive effort and oxidative damage are also observed.

\section{MATERIALS AND METHODS}

\section{(1) Literature search and criteria for inclusion in the meta-analysis}

Data for the meta-analysis were collated from the literature and from additional unpublished sources. The search was initiated using recent reviews (Metcalfe \& Monaghan, 2013; Selman et al., 2012; Speakman \& Garratt, 2014; Stier et al., 2012), followed by additional searches using Web of Science and Google Scholar up to 15 May 2014. We used the search terms "reproduction", "reproductive effort", "oxidative damage", "oxidative stress" and their combinations. These searches yielded 1,672 articles (including duplicate results); based on a reading of abstracts alone this list was reduced to 44 studies, which were read in full. The vast majority of studies of relationships between reproduction and oxidative damage have reported data for homeothermic species, and for females only. Therefore, we restricted our search to females of homeothermic species. We sought data for two types of analysis. First, we were interested to test the hypothesis that individuals expending greater reproductive effort have 
higher levels of oxidative damage (i.e. an analysis across females that have all reproduced, but have variable numbers or weights of offspring). For this analysis we collated data for the association between reproductive effort (i.e. clutch, brood or litter size; litter mass; or the proportion of eggs that hatched) and oxidative damage. Second, we were interested to test the hypothesis that levels of oxidative damage are higher in reproductive than in non-reproductive individuals; here we collated data for categorical comparisons of breeders versus non-breeders from the same populations or social groups.

We collated data for five markers which are commonly reported as indicators of oxidative damage: malondialdehyde, reactive oxygen metabolites, protein carbonyls, oxidised glutathione, and protein thiols (Halliwell \& Gutteridge, 1999; Monaghan et al., 2009; Selman et al., 2012). Malondialdehyde is a marker of lipid peroxidation which is quantified using either high-performance liquid chromatography (HPLC) or spectrophotometry; the latter method has lower specificity and accuracy than HPLC, but there is no reason to expect this to bias the results of the meta-analyses. Reactive oxygen metabolites are hydroperoxides, generated mainly as a result of lipid peroxidation. Protein carbonyls are produced when protein side chains become oxidised, and are therefore a marker of protein oxidation. Oxidised glutathione is a marker of redox status, and in some instances oxidative stress. The above markers were predicted to increase with reproduction. In addition, we collated data for protein thiols, groups on proteins that are susceptible to oxidation and are therefore a marker of protein oxidation. Reproduction is predicted to diminish levels of protein thiols, but for the purposes of the meta-analysis we reversed the sign of these data so all five markers of oxidative damage were predicted to increase with reproduction. This enabled us to calculate combined effect sizes across tissues and/or markers (see Section III, 2, below). Data were available for blood plasma, serum and 
erythrocytes, skeletal muscle, liver, kidney, heart, and ovarian follicles (see online supporting information, Tables S1 and S2).

If samples were collected at different times during reproduction, we used data for the sampling point that represented peak reproductive effort (peak lactation rather than weaning; Upreti, Chaki \& Misro, 2002). In two studies of cooperatively breeding bird species, data were available for the period just prior to laying, during offspring care, and/or at completion of breeding (Cram, Blount \& Young, 2014; van de Crommenacker, Komdeur \& Richardson, 2011). In such species, subordinate 'helpers' often contribute to nestling care, and sometimes also engage in independent breeding attempts. Therefore in order to compare breeders against individuals that were functionally non-reproductive, we used data collected just prior to laying for dominant females and non-reproductive subordinates. For studies that experimentally manipulated a variable, i.e. ambient temperature, diet, or radiofrequency radiation (RF) exposure, in addition to measuring reproduction, we used data for the control group [i.e. room temperature $\left(21^{\circ} \mathrm{C}\right)$; non-supplemented diet; non RF-exposed] (Fletcher et al., 2012; Tomruk, Guler \& Dincel, 2010; Yang et al., 2013). Similarly, one study included data for a line of animals selected for high metabolic rate; we used data for the non-selected (control) line (Oldakowski et al., 2012). For one other study, data were available for different age classes; we used data for individuals at peak fecundity (da Silva et al., 2013).

We excluded certain types of studies in order to avoid sources of bias that could confound the meta-analyses. These included studies of Bos taurus (dairy cows); such animals have been selected for extreme reproductive output, and these studies invariably fail to include an appropriate control (non-breeding) comparator (Metcalfe \& Monaghan, 2013). Two other studies were excluded because the effect of reproduction on oxidative damage could not be 
separated from a potential influence of environment (wild breeders compared with captive nonbreeders) (Casagrande et al., 2011), or because data were pooled for both sexes (Beaulieu et al., 2011). Finally, we excluded one study of Rattus norvegicus (Sprague Dawley rats), because null results were mentioned in the text but details were not provided, and therefore effect sizes could not be calculated for these variables (Sainz et al., 2000).

\section{(2) Data extraction and calculation of effect sizes}

Data for the analysis of oxidative damage in relation to reproductive effort were extracted from the text and tables in the form of Pearson's correlation coefficients, or slope estimates (and standard errors) from general linear models, sample sizes, and $P$-values. We also used dichotomous data in the form of means ( \pm S.D.) for the comparison of individuals expending relatively high versus lower reproductive effort. Effect sizes for this diverse data set were calculated as described below. Similarly, data for the analysis of oxidative damage in breeders compared with non-breeders were extracted from the text and tables in the form of means ( \pm S.D.) and sample sizes, or from graphs using GSYS version 2.4 graph digitising software (Nuclear Reaction Data Centre, Hokkaido University; www.jcprg.org/gsys/). For the following papers we requested and received additional information from the authors: Alonso-Alvarez et al. (2010); Bergeron et al. (2011); da Silva et al. (2013); Garratt et al. (2013); King, Garratt \& Brooks (2013); Markó et al. (2011); Nussey et al. (2009); Xu et al. (2014). One study presented data in the form of medians, ranges and sample sizes (da Silva et al., 2013); we estimated means ( \pm S.D.) using standard formulae (Hozo, Djulbegovic \& Hozo, 2005).

Statistics were converted to effect sizes in the form of Hedges' $g$, using Comprehensive Meta-Analysis (CMA) software (Borenstein et al., 2005). Hedges' $g$ is a measure of bias- 
corrected standardised mean difference which can be generated from categorical or continuous data (Hedges, 1981), and is therefore appropriate for diverse data sets. The data set used for analysis of oxidative damage in relation to reproductive effort included both continuous and dichotomous data as described above. Using Hedges' $g$ as a standard measure of effect size allowed us to compare both qualitatively and quantitatively the differences in oxidative damage linked to breeding per se, and linked to relatively high versus lower reproductive effort. To infer the relationship between reproductive effort and oxidative damage on continuous scales, and to verify that results of the meta-analysis for reproductive effort were not biased by the method used for calculation of effect sizes, we also calculated Fisher's $Z$ for all data points in reproductive effort analyses. Fisher's $r$-to- $Z$ transformation provides a variance-adjusted effect size, typically used to describe continuous data.

\section{(3) Procedures for meta-analysis}

Data were analysed in CMA (Borenstein et al., 2005). Random-effects meta-analysis models were used, which weight data points according to a study sampling variance that is calculated based on the assumption that within-study sampling variance and between-study sampling variance are independent of one another (Borenstein et al., 2010). This assumption is more accurate and more conservative compared to fixed-effects meta-analysis models, which assume that within- and between-study variances are equal, when analysing multiple data sets from different species. A tiered approach to analysis was taken: first, all tissues and all markers were analysed simultaneously. If this analysis showed $P \geq 0.05$ for the summary effect size, each tissue was then analysed independently to ascertain whether there were significant consistencies across markers within tissues. If this showed no significance ( $P \geq 0.05$ for the summary effect 
size), then subsets of data for each individual tissue-marker combination were analysed, to ascertain whether specific markers within specific tissues showed significant consistencies in relation to reproduction. In 'all-tissues-all-markers' models, and 'individual-tissues-all-markers' models, where a study included data for multiple tissues and/or markers, the combined effect size was used. Similarly, where a study had reported both data from observational analyses and from experimental manipulation, the combined effect size was used.

Shared evolutionary history can potentially bias the results of meta-analyses (Chamberlain et al., 2012). CMA is unable to scale models according to relatedness metrics, so in order to test the importance of phylogenetic influence we re-ran the analyses carried out in CMA, utilising MCMCglmm (Hadfield, 2010) in the R programming language (R Development Core Team, 2014). None of these models indicated an important phylogenetic component (see Appendix S1). A phylogeny should not be included in an analysis when there is little or no support for a phylogenetic effect, as forcing a relatedness-based variance-covariance matrix in the random effects where there is no evidence to support the existence of an important influence of phylogenetic relatedness has the consequence of increasing credible intervals on fixed-effects estimates, and therefore increasing the likelihood of type II error. Therefore, results presented in the main text are derived from CMA.

\section{(4) Evaluating potential sources of bias in the meta-analysis}

Publication bias, i.e. the selective reporting of statistically significant results, can be a problem for meta-analyses (Borenstein et al., 2005; Sterne et al., 2005; Koricheva et al., 2014). We tested for evidence of publication bias using two methods: funnel plot, and trim-and-fill. Funnel plots represent a measure of study size (e.g. standard error) on the $y$-axis as a function of effect size on 
the $x$-axis; clustering of studies on one side of the mean or the other indicates bias, reflecting the fact that smaller studies (that are plotted towards the bottom) are more likely to be published if they have above-average effect sizes. Trim-and-fill is a method that trims any asymmetric studies from a funnel plot in order to calculate the unbiased effect size, and then fills the plot by reinserting the trimmed studies in addition to imputed counterparts to attain symmetry. Funnel plots are unlikely to be useful where there are fewer than $N=5$ studies in a meta-analysis (Sterne et al., 2005), and therefore we used funnel plots and trim-and-fill in all instances where there were five or more studies. Results of these analyses are shown in Appendix S2.

Where existing studies have failed to detect an oxidative cost of reproduction, this might be because they have focussed on laboratory-housed animals rather than individuals living in natural populations. In addition, individuals that reproduce are unlikely to be a random subset of a population; breeders may be younger, higher quality or in better physiological condition than non-breeders. Therefore, studies might have failed to detect an oxidative cost of reproduction if they have relied on observational evidence, rather than having drawn animals from the same populations or social groups and randomly allocated them to breeding and non-breeding conditions (Metcalfe \& Monaghan, 2013; Selman et al., 2012; Speakman \& Garratt, 2014). Also, individuals may modulate their reproductive effort as a function of individual quality or condition; experimental manipulation of reproductive effort may therefore be necessary to detect an oxidative cost of reproduction (van Noordwijk \& De Jong, 1986; Metcalfe \& Monaghan, 2013). We evaluated whether there was any such evidence of systematic bias, by comparing the results of the full meta-analysis with results of meta-analyses that excluded different subsets of data in turn (laboratory studies; field studies; observational studies; studies that had not manipulated reproductive effort) (see Appendix S2). 
Heterogeneity analysis is a means of assessing the consistency in effect sizes across studies; significant heterogeneity may arise for example where there are interspecific differences in effect sizes that reflect the biology of species, or where there are methodological differences amongst studies (Koricheva et al., 2014). Heterogeneity analyses were carried out using CMA software, in terms of Cochran's $Q$ which is calculated by summing the squared deviations of each study's estimate from the overall meta-analytic estimate, weighting each study's contribution as in the meta-analysis. $P$ values are calculated using a $\chi^{2}$ distribution with $k-1$ degrees of freedom (where $k$ is the number of studies). In addition, we calculated $I^{2}[100 \% \times(Q-$ d.f. $) / Q]$ which describes the proportion of total variation in studies estimates that is due to heterogeneity (Higgins \& Thompson, 2002).

\section{RESULTS}

\section{(1) Reproductive effort and oxidative damage}

Available data for the association between reproductive effort and oxidative damage came from eight studies of five species of mammals, and from three studies of three species of birds (Alonso-Alvarez et al., 2010; Bergeron et al., 2011; Costantini et al., 2014a; Garratt et al., 2013, 2011; King et al., 2013; Markó et al., 2011; Nussey et al., 2009; Stier et al., 2012; Xu et al., 2014; Yang et al., 2013) (Table S1). Consistent with the predicted pattern, across all tissues and markers there was a significantly higher level of oxidative damage in mothers with relatively high compared with lower reproductive effort (Fig. 1). This result was qualitatively the same when effect sizes were calculated using Fisher's $Z$ (random effects meta-analysis: Fisher's $Z=$ $0.176 ; 95 \%$ confidence interval: lower limit, 0.047 , upper limit, $0.305 ; \mathrm{Z}=2.667, P=0.008$ ). As Fisher's $\mathrm{Z}$ is a variance-adjusted form of a correlation coefficient, we can conclude from this that 
there is a continuously increasing relationship between offspring number and oxidative damage from relatively low to higher reproductive effort.

Markers of oxidative damage in blood plasma, serum, or erythrocytes were measured by nine of the studies included in the analysis, while four studies had measured such markers in organs (Table S1). We therefore examined whether the association between reproductive effort

and oxidative damage held when considering blood and organ markers independently. Across all blood markers there was a significant positive association between reproductive effort and oxidative damage (Hedges' $g$ : 0.341; 95\% confidence interval: lower limit, 0.043 , upper limit, $0.639 ; \mathrm{Z}=2.245, P=0.025)$, whereas across all organ markers while the sign of the effect size was positive, no significant pattern existed (Hedges' $g$ : $0.231 ; 95 \%$ confidence interval: lower limit, -0.242 , upper limit, $0.704 ; \mathrm{Z}=0.957, P=0.338$ ). Therefore, the positive association between reproductive effort and oxidative damage was largely driven by variation in levels of blood markers.

\section{(2) Reproductive state and oxidative damage}

To test the hypothesis that reproducing individuals have increased levels of oxidative damage, we next made categorical comparisons of breeders versus animals drawn from the same populations or social groups which had not bred. Available data for this comparison came from 15 studies of 11 species of mammals, and from three studies of three species of birds (Bergeron et al., 2011; Cederberg, Basu \& Eriksson, 2001; Costantini et al., 2014b; Cram et al., 2014; da Silva et al., 2013; Fletcher et al., 2012; Garratt et al., 2013, 2011; Jan et al., 2014; Oldakowski et al., 2012; Schmidt, Blount \& Bennett, 2014; Stier et al., 2012; Tomruk et al., 2010; Upreti, Chaki \& Misro, 2002; van de Crommenacker et al., 2011; Xu et al., 2014; Yang et al., 2013), 
and additional unpublished data from a study of banded mongooses (Mungos mungo) (Table S2). In a meta-analysis including all tissues and markers, there was no consistent association between reproduction and oxidative damage (Fig. 2). We therefore examined whether there was consistency across all markers of oxidative damage for each individual tissue type, for those tissues where multiple markers were available. Again, we found no consistent patterns for any tissue (Fig. 2). This was because, opposite to the predicted relationship, levels of malondialdehyde and protein carbonyls in heart were significantly lower in breeders compared with non-breeders. Oxidised glutathione and protein thiols in heart did not vary consistently with reproductive state (Fig. 2). Similarly, levels of protein carbonyls in ovarian follicles were markedly and significantly lower in breeders compared with non-breeders (Fig. 2). In liver, malondialdehyde and oxidised glutathione showed considerable variation amongst studies and no consistent patterns, whereas protein carbonyls and protein thiols indicated markedly and significantly decreased levels of oxidative damage in reproductive compared to non-reproductive animals (Fig. 2). Skeletal muscle and kidney showed no consistent patterns for any marker of oxidative damage in relation to reproduction (Fig. 2).

In contrast to data for organs, blood markers of oxidative damage were borderline significantly higher in breeders compared with non-breeders in an analysis across all markers. This was because blood levels of protein carbonyls were markedly and significantly higher in breeders compared with non-breeders. However, levels of malondialdehyde, reactive oxygen metabolites and protein thiols showed no consistent patterns in relation to reproduction (Fig. 2).

There was no evidence to suggest that these results are confounded by publication bias, or by systematic bias due to data that were derived from laboratory studies, or field studies, or observational evidence (i.e. where animals had 'self-selected' whether to breed), or experiments 
that had not manipulated reproductive effort, respectively (see Appendix S2). Surprisingly, however, the conclusion that reproductive effort is positively associated with oxidative damage was not changed in an analysis excluding data for manipulated reproductive effort (Hedges' $g$ : 0.361; $95 \%$ confidence interval: 0.047 and $\left.0.674 ; \mathrm{Z}=2.254, N_{\text {studies }}=9, P=0.024\right)$. By contrast, excluding all correlational data, i.e. data for breeders that had not experienced a manipulation of reproductive effort, rendered the relationship between reproductive effort and oxidative damage non-significant (Hedges' $g$ : $0.123 ; 95 \%$ confidence interval: -0.262 and $0.509 ; \mathrm{Z}=0.627, N_{\text {studies }}$ $=4, P=0.531 ;$ Table S4).

There was significant heterogeneity in effect size for several of the meta-analyses comparing breeders and non-breeders (Table 1).

\section{DISCUSSION}

Consistent with theory, using meta-analysis we found a significant positive association between reproductive effort and oxidative damage across tissues and markers. However, opposite to the pattern predicted based on existing theory, categorical comparisons of breeders versus nonbreeders revealed that reproduction is associated with significant reductions in certain markers of oxidative damage in heart, liver and ovarian follicles. Fig. 3 summarises these main findings of the meta-analysis schematically. Patterns of oxidative damage in other tissues showed no statistically significant associations with reproduction (Fig. 2). Blood protein carbonyls showed a contrasting pattern, levels being significantly increased in breeders compared with non-breeders, while other blood markers did not differ significantly in association with reproductive state. Sample sizes were small for many of these comparisons, meaning that statistical nonsignificance can in many cases be attributed to a lack of power. Indeed, using Cohen's benchmarks for interpreting effect sizes, i.e. Hedges' $g$ of $0.2,0.5$, and 0.8 , as small, medium, 
and large, respectively (Cohen, 1988), and considering data for all organ tissues and individual markers, we find that there are eight large, three medium and two small effect sizes, while only two effect sizes can be considered trivial. All but one of these effect sizes are negatively signed. Considering data for individual markers in blood, we find that there are two large positively signed effect sizes, one medium negatively signed effect size, and one trivial (positively signed) effect size.

The reduction in oxidative damage in breeders is not simply because reproducing animals were in better physiological condition or were younger; the same pattern was observed when including only data from experiments that randomly allocated individuals to treatment groups (see Appendix S2). Such experiments typically confirmed that females allocated to breeding and non-breeding groups were of similar body mass and age (da Silva et al., 2013; Garratt et al., 2013, 2011; King et al., 2013; Pichaud et al., 2013; Stier et al., 2012; Tomruk et al., 2010; Yang et al., 2013), and therefore confounding biases in oxidative damage would not be expected. These consistent but contrasting relationships between oxidative damage and reproductive effort, versus the reproductive state per se, may help explain why many existing studies have concluded that reproduction has little or no oxidative cost. Rather, we hypothesise that the cost of reproduction could be mediated by dual impacts of oxidative damage on mothers and offspring, while mothers may face additional costs diminishing such damage.

Since our meta-analysis was based on data for multiple tissues and markers of oxidative damage, and several different species of mammals and birds, heterogeneity in effect sizes is to be expected. Values for $I^{2}$ of $25 \%, 50 \%$, and $75 \%$, represent low, medium, and high levels of heterogeneity, respectively (Higgins et al., 2003). Indeed, values for Cochran's $Q$ and $I^{2}$ show that there is significant and marked heterogeneity in effect sizes amongst studies for some tissue- 
marker combinations (Table 1). Such heterogeneity is likely to arise because of variation in the biology of different species, but also methodological differences amongst studies (e.g. Costantini \& Møller, 2008). For example, most studies in the meta-analyses that reported malondialdehyde as a marker of oxidative damage used a spectrophotometric assay that has been criticised for lack of specificity (reviewed by Monaghan et al., 2009; Selman et al., 2012), while a few recent studies have used a more precise HPLC method (e.g. Cram et al., 2014; Schmidt et al., 2014). Across all tissues and all markers, effect sizes were fairly consistent $\left(I^{2}=28 \%\right)$ for the relationship between reproductive effort and oxidative damage, while having a high level of heterogeneity $\left(I^{2}=81 \%\right)$ for the association between reproductive state and oxidative damage. The reason for this difference is unclear, but it may simply be because most data relating to reproductive effort were for blood markers, whereas data relating to reproductive state were for a wide variety of tissues. The existence of significant heterogeneity highlights the importance of using random-effects meta-analyses when analysing multiple data sets from different species. Random-effects models are relatively conservative because they do not assume homogeneity in effect size across studies, an assumption that is unlikely to hold when comparing results from multiple species or methodological approaches (Borenstein et al., 2010).

\section{(1) Why is oxidative damage reduced in reproducing females?}

We hypothesise that transition to the reproductive state triggers a pre-emptive reduction in levels of oxidative damage in certain markers and tissues, in order to shield mothers, and in particular their gametes and developing offspring, from harm caused by an inevitable increase in oxidative damage resulting from expenditure of reproductive effort. If, as we hypothesise, such preemptive reductions in oxidative damage serve to shield mothers and offspring from oxidative 
insults during sensitive periods of reproduction, the timing of such 'oxidative shielding' is likely to differ amongst taxa. We now discuss the hypothesised importance of oxidative shielding in relation to maternal life histories, follicular growth, placental function and lactation in eutherian mammals, and in relation to egg production in oviparous species.

\section{(a) Oxidative shielding in eutherian mammals}

Reproductive effort is positively correlated with oxidative damage (Fig. 1). Mothers might therefore gain from pre-emptive reduction of oxidative damage in preparedness for reproduction, to reduce the oxidative costs of a given level of reproductive effort, or to ensure that levels of oxidative damage do not subsequently exceed some critical threshold above which it may be impossible to maintain cellular homeostasis. For example, uncontrolled oxidative stress arising from reproductive effort could result in the proteolytic capacity of cells becoming overwhelmed by large numbers of oxidized protein molecules, leading to impaired proteolytic digestion. Rather than being broken down, such oxidized proteins may instead become aggregated, and impair cell and organ function (Davies, 2000).

We hypothesise that reductions in oxidative damage in reproducing females may also serve to shield gametes and developing offspring. In placental mammals, oxygen tension is an important regulator of trophoblast cell proliferation and differentiation (Bose et al., 2006). During early pregnancy, ROS regulate various cell functions and physiological hypoxia of the gestational sac is thought to protect the foetus from oxidative damage (Bose et al., 2006; Jauniaux, Poston \& Burton, 2006). This is supported by animal studies which have demonstrated increased blastocyst developmental rate at lower oxygen tension (Gupta et al., 2007). However, at the onset of embryonic aerobic metabolism, induced by the establishment of utero-placental 
blood flow, oxygen tensions inside the intervillous space increase leading to a burst of oxidative stress (Al-Gubory, Fowler \& Garrel, 2010; Gupta et al., 2007). High levels of placental and systemic oxidative stress in mothers have been linked to altered placental vasculature, defective embryogenesis and pregnancy loss (Al-Gubory et al., 2010; Gupta et al., 2007). One mechanism linked to pregnancy loss in humans is antiphospholipid (aPL) antibody syndrome: oxidative damage to low density lipoproteins (LDLs) can alter the antigenic properties of modified phospholipids resulting in formation of aPL antibodies directed against them (Gupta et al., 2007; Vial et al., 2013). In laboratory rodents, up to $10 \%$ of pregnancies fail post-implantation (Wilmut, Sales \& Ashworth, 1986). Comparable data are limited for other taxa, and non-existent for natural populations of animals (Jauniaux et al., 2006). In order to assess the potential adaptive significance of oxidative shielding, it would be valuable to gather more data on associations between oxidative stress and pregnancy outcomes in a broader range of species, including studies in the wild.

Sub-lethal effects of placental oxidative stress on embryos are also likely to be very important, and have been highlighted as a potential general mechanism linking altered placental function to life-history trajectories, i.e. 'fetal programming' (Myatt, 2006). For example, placental dysfunction caused by oxidative stress has been linked to fetal growth restriction and low birth weight in humans (Al-Gubory et al., 2010; Gupta et al., 2007). Studies of natural populations of mammals have shown that conditions experienced in natal environments can impact on reproduction and survival in later life (Bouwhuis et al., 2010; Nussey et al., 2007). Oxidative stress is a candidate mechanism underlying such effects (Isaksson et al., 2011), but we are not aware of any studies that have examined links amongst oxidative stress, placental 
dysfunction and life-history trajectories in any non-human animal species. This seems likely to be a worthwhile direction for future research.

Lactation imposes huge nutritional demands on mothers: organs such as the liver and alimentary tract grow, while stored reserves are catabolised (Hammond et al., 1994; Speakman, 2008) which increases ROS production and the risk of oxidative stress (Schaff et al., 2012). Oxidative stress in lactating females can have several deleterious impacts on offspring, for example by reducing milk yield (Chandra et al., 2013; Suriyasathaporn et al., 2009), and by impairing colostrum and milk quality in terms of antioxidant content (Rizzo et al., 2013a). At parturition offspring are at risk of oxidative stress as they are exposed to atmospheric concentrations of oxygen; antioxidant enzymes and vitamins in colostrum and milk therefore confer protection against oxidative stress during neonatal development (Albera \& Kankofer, 2009; Castillo et al., 2005; He et al., 2008). Finally, malondialdehyde (MDA) is present in milk (Rizzo et al., 2013b; Suriyasathaporn et al., 2009; Wicheansoni et al., 2007), and levels of MDA in maternal blood and milk are correlated in dairy cows (Bouwstra et al., 2008). MDA is highly reactive and may therefore initiate lipid peroxidation in milk and reduce its nutritional quality. Furthermore, ingestion of oxidised fats and MDA in milk could cause lipid peroxidation in vivo and be detrimental to offspring health (Serini et al., 2011). We therefore hypothesise that mothers are selected to shield offspring from such effects by reducing levels of oxidative damage during lactation.

The majority of studies of mammals used in the meta-analysis comparing breeders and non-breeders presented data for lactating females, whereas three studies presented data for females during pregnancy, and one study presented data for ovarian follicles (see Table S2). We hypothesise that levels of oxidative damage will be consistently reduced from oocyte maturation 
through gestation and lactation in mammals, compared to non-breeding periods. However, to the best of our knowledge, no studies to date have measured oxidative damage levels in the same individuals during pre-breeding, pregnancy, lactation, and again post-breeding for any animal species. Collection of these kinds of longitudinal data would be extremely valuable to ascertain whether reductions in oxidative damage are of similar or different magnitude across these distinct stages of reproduction.

\section{(b) Oxidative shielding in oviparous animals}

Our meta-analysis comparing breeders and non-breeders also included data for egg-laying females of three species of birds (see Table S2). In oviparous species, the egg provides all the nutrients required for embryonic development; oxidative stress could be deleterious for the production and viability of eggs if it resulted in depletion of lipids and proteins, or antioxidants (carotenoids, and vitamins $\mathrm{A}$ and $\mathrm{E}$ ) that are required for albumen and yolk synthesis (Blount, Houston \& Møller, 2000). In addition, oxidative stress in females during egg production has the potential to result in damage to specific proteins, such as immunoglobulins that are required to confer passive immunity for offspring (Haq, Bailey \& Chinnah, 1996), and lysozymes that confer anti-bacterial defence in eggs. Furthermore, aldehydic lipid peroxidation products such as malondialdehyde are incorporated into the lipid matrix of yolk (Mohiti-Asli et al., 2008; Mourente et al., 1999), which may cause oxidative degeneration of yolk nutrients (Grune et al., 2001), and lipid peroxidation in vivo. Studies of natural populations of birds have shown that females with greater resistance to oxidative stress produce larger clutches with higher hatching success (Bize et al., 2008), and have linked conditions experienced in the natal environment to adult reproduction and senescence (Cartwright et al., 2014; Millon et al., 2011). It would 
therefore be interesting to examine how maternally-derived oxidative damage in eggs may affect offspring. To date, no study has examined whether levels of oxidative damage in mothers and their eggs and offspring are correlated, or how maternally-derived oxidative damage may influence the life histories of offspring.

In oviparous animals which reproduce only once in a single season, we hypothesise that levels of oxidative damage will be consistently reduced in mothers during conception and egg production, compared to incubation, provisioning or non-breeding periods. For species that lay multiple times over a single breeding season, it would be interesting to examine whether any decrease in levels of oxidative damage is maintained throughout all phases of reproduction, or alternatively, whether oxidative damage is diminished only during conception and egg production. The latter may be expected, because oxidative shielding presumably incurs costs (otherwise females would maintain low levels of oxidative damage all of the time). Finally, for oviparous mammals (i.e. monotremes) that lactate, or birds that provision their young with crop secretions (e.g. columbids), it would be interesting to investigate whether oxidative damage products are found in these fluids, and if so, whether levels of oxidative damage are decreased in mothers while they provision dependent offspring.

\section{(2) Consistency and inconsistency amongst markers of oxidative damage}

It has previously been emphasised that levels of oxidative damage may vary amongst specific markers and tissues, underlining the importance of measuring multiple markers and (if possible) different tissues when studying oxidative stress (reviewed in Costantini, 2008; Selman et al., 2012). The meta-analysis results support this view, in so far as categorical comparisons of breeders and non-breeders revealed tissue- and marker-specific associations between 
reproduction and oxidative damage. In breeders, oxidative damage was significantly reduced in liver and heart - metabolically active organs that increase in mass during lactation, presumably to optimise processing of nutrients and elimination of waste products (Hammond et al., 1994). Levels of oxidative damage were also significantly decreased in ovarian follicles from cyclic compared with acyclic water buffalos (Bubalus bubalis) (Jan et al., 2014). ROS have important signal functions during follicular development, but are also responsible for initiation of apoptosis in primordial, primary and antral follicles, whereas antioxidants protect against oxidativedamage-induced follicular apoptosis (Gupta et al., 2007; Jan et al., 2014). In addition, oxidative damage to germline DNA could result in genetic changes being transmitted to offspring via common genetic inheritance (mutations) and/or epigenetic inheritance, with deleterious consequences for offspring fitness (Velando, Torres \& Alonso-Alvarez, 2008). Oxidative shielding in these organs is therefore likely to have an important role in maintaining functions which are vital for successful reproduction and the production of high-quality offspring.

The significant positive association between reproductive effort and oxidative damage was largely driven by data for various blood markers (Fig. 1), while in stark contrast to data for organ markers, categorical comparison revealed that blood protein carbonyls were significantly higher in reproductive compared with non-reproductive females (Fig. 2). This suggests that blood markers provide a signature of current reproductive effort, which organ markers do not provide over equivalent, short timescales. It would therefore be interesting to assess whether blood markers of oxidative damage are decreased upon transition to the reproduction state, in line with patterns observed for organs.

Why blood protein carbonyls in particular are significantly increased in breeders is unclear. One possibility is that increased protein turnover in reproducing animals results in 
greater availability of substrates that are susceptible to carbonylation. Alternatively, carbonylation may serve an important 'protein quality control' function, signalling mistranslated or otherwise aberrant proteins for proteolytic degradation to prevent their incorporation into biomolecules involved in information transfer (e.g. ribosomes; RNA and DNA polymerases), while also yielding amino acids for de novo protein synthesis (Nystrom, 2005). To help separate these possibilities, it would be interesting to examine whether blood levels of protein carbonyls correlate positively or negatively with reproductive success. Ultimately, to improve our understanding of the role played by oxidative stress in life-history evolution, we need more studies that link variation in individual markers of oxidative damage to functional outcomes, particularly reproductive success and survival (Metcalfe \& Monaghan, 2013; Selman et al., 2012). Such evidence is still remarkably scarce (see for example Noguera, Kim \& Velando, 2012).

\section{(3) How is oxidative damage reduced in reproducing females?}

Oxidative damage could be decreased by various potential mechanisms. An obvious possibility is via increased levels of antioxidant defences. Consistent with this explanation, studies have linked decreased levels of lipid and protein oxidation to increased total glutathione (tGSH) and total superoxide dismutase (SOD) activity in the liver of reproducing wild-derived house mice (Mus musculus domesticus) (Garratt et al., 2013, 2011), increased liver SOD and non-enzymatic antioxidant activity (total antioxidant capacity, TAC) in lactating Mongolian gerbils (Meriones unguiculatus) (Yang et al., 2013), and increased liver SOD activity in lactating Brandt's voles (Lasiopodomys brandtii) (Xu et al., 2014). Such increased activity of antioxidant defences could be triggered by changes at the cellular, mitochondrial level (Pichaud et al., 2013). Lactating mice 
have lower electron transport system activity and higher mitochondrial density in liver compared with non-breeders, which could in turn lead to upregulated antioxidant defences (Pichaud et al., 2013).

A review of the broader literature including studies of birds reveals that relationships between reproduction and antioxidant defences and/or resistance to oxidative stress are often contradictory (Stier et al., 2012). Indeed, several studies of birds have reported that blood levels of antioxidant defences are decreased during reproduction (Alonso-Alvarez et al., 2004; Losdat et al., 2011; Wiersma et al., 2004). This apparent discrepancy among taxa might be because existing studies of mammals have measured antioxidant defences in various tissues, sometimes including blood, whereas studies of birds have invariably measured antioxidant defences in blood only. Across taxa, there is a tendency for increased blood levels of oxidative damage in breeders (Fig. 2). In breeding birds, it has been argued that reduced blood levels of antioxidant defence represent a cost of reproduction, i.e. the defences are sacrificed (Alonso-Alvarez et al., 2004; Wiersma et al., 2004; Losdat et al., 2011). Alternatively, it seems possible that such reductions in antioxidant defences might allow increased levels of oxidative damage to circulating macromolecules, specifically in order to yield e.g. amino acids for de novo synthesis of nutrients (discussed above; see Section V, 2). Ultimately, to separate these possibilities we will need greater replication in measurements of specific markers of oxidative damage across tissues and taxa, coupled with studies of how variation in specific markers correlates with fitness.

A decrease in oxidative damage in breeders might also be explained by uncoupling proteins (UCPs), reducing ROS production in mitochondria (Brand, 2000). However, UCP gene expression is downregulated or unchanged during lactation (Speakman, 2008), making mitochondrial uncoupling an unlikely general explanation for decreased oxidative damage in 
breeders. Moreover, mitochondrial uncoupling seems an unlikely mechanism by which animals would decrease levels of oxidative damage during reproduction, because this would result in reduced efficiency of energy production at a time when metabolic demands are likely to be particularly high. Additional studies are required to assess whether uncoupling is associated with decreased oxidative damage during other stages of reproduction, in other tissues, and in other taxa. Decreased levels of oxidative damage in breeding females might further be explained by the action of oestradiol. Studies of rats have shown that ovariectomy leads to increased oxidative damage, whereas oestrogen administration increases levels of glutathione and glutathione peroxidase (GPx) activity, and results in decreased lipid peroxidation in various tissues (Aksakal et al., 2011; Borras et al., 2003; Kireev et al., 2007; Persky et al., 2000). In vitro experiments have confirmed that oestradiol acts by binding to oestrogen receptors and subsequently activates the mitogen activated protein (MAP) kinase and nuclear factor kappa B (NF- $\mathrm{kB}$ ) signalling pathways, causing increased expression of genes that code for antioxidants such as SOD and GPx (Vina et al., 2005).

We have hypothesised that reduced oxidative damage in certain tissues of reproducing females is an adaptive response to enhance reproductive success. However, we must consider the alternative possibility that a simple dilution effect might explain reduced levels of oxidative damage in tissues such as heart, liver and ovarian follicles, which become enlarged during reproduction. As previously argued, dilution seems an unlikely explanation for decreased oxidative damage in breeders, because: $(i)$ not all markers of oxidative damage in such tissues are significantly lower in breeders compared with non-breeders, see Fig. 2; (ii) the fact that certain antioxidants have been measured at higher levels in such tissues in breeders is inconsistent with a dilution effect (Garratt et al., 2013, 2011; Xu et al., 2014; Yang et al., 2013); 
and (iii) blood volume also increases in lactating mammals, but there is little or no indication that blood markers of oxidative damage are reduced in breeders (Yang et al., 2013; and see Fig. 2).

\section{(4) How costly is the diminishment of oxidative damage?}

To what extent diminishment of oxidative damage is costly for breeding females is an important question, because costs of reproduction are likely mediated both by oxidative damage and by mechanisms to minimise or repair such damage (Monaghan et al., 2009; Selman et al., 2012). Such costs may depend on the mechanism responsible for decreased oxidative damage. For example, since antioxidant defences have low energetic costs, it has been argued that nutrition per se is unlikely to limit protection against oxidative damage (Speakman \& Garratt, 2014). However, the efficiency of energy production is decreased by mitochondrial uncoupling (Brand, 2000), which could hypothetically lead to energy limitation of foraging capacity, and therefore reduced ability to sequester resources required to initiate or sustain reproductive effort. Such resources could include macronutrients, but also micronutrients such as vitamins $\mathrm{A}, \mathrm{C}$ and $\mathrm{E}$, flavonoids, and carotenoids, which may themselves be limiting for reproduction and potentially also antioxidant defence (Monaghan et al., 2009). Alternatively, at physiological levels ROS have important roles in various signalling transduction pathways (Al-Gubory et al., 2010; Metcalfe \& Alonso-Alvarez, 2010), and therefore if oxidative shielding reduces ROS production this could impact on diverse aspects of cell and organ function.

If reducing oxidative damage is costly, mothers might be selected to show graded rather than all-or-nothing reductions in oxidative damage on transition to the reproductive state, proportional to the scale of their reproductive investment (i.e. offspring number). Recent research on house mice has found significant differences in mitochondrial function and density 
in reproductive compared with non-reproductive females, while there were no such differences in relation to experimentally manipulated litter size (Pichaud et al., 2013). This could indicate that physiological restructuring associated with reproduction, e.g. in mitochondria, represents an all-or-nothing transition, rather than varying in relation to reproductive effort (Pichaud et al., 2013). However, experimental manipulations of offspring number are usually carried out postbirth, by which time mothers have already undergone extensive physiological restructuring, and further rapid modification may not be possible within the remaining period of parental care.

It has recently been argued that the ability to detect an oxidative cost of reproduction could critically depend on experimental manipulation of brood or litter size, to overcome the potential problem that individuals may adjust their reproductive effort to their own quality or access to resources (Metcalfe \& Monaghan, 2013). However, we found no consistent evidence to suggest that the results of the meta-analysis were confounded by studies that have failed to manipulate reproductive effort. On the contrary, the conclusion that reproductive effort is positively associated with oxidative damage was not changed in an analysis based on correlational data only, i.e. excluding data for manipulated reproductive effort. By contrast, excluding data for breeders that had not experienced a manipulation of reproductive effort rendered this relationship non-significant (Table S4). We can suggest three, non-mutually exclusive possible explanations for this discrepancy between correlational and experimental data. Firstly, females may not be able to readjust their physiology rapidly to the altered workload imposed by experimental manipulation of offspring number when it is carried out post-birth, as discussed above. If reductions in oxidative damage in breeding females are graded, it seems possible that the degree of reduction is set when initial decisions about offspring number are made (i.e. at follicle enlargement and ovulation). To test this idea experimentally would require 
manipulation of follicle number, for example by surgical intervention (e.g. Sinervo et al., 1990), or via egg removal and egg addition manipulations in oviparous species that are indeterminate layers.

Secondly, we must consider the possibility that individuals with relatively high baseline (i.e. pre-breeding) levels of oxidative damage subsequently produce more offspring. It is clear that metabolic rate often increases during the early stages of reproduction, for example due to costs of egg production in birds (e.g. Vézina \& Williams, 2002). Such increased metabolic rate in early reproduction may be expected to incur an oxidative cost, and hence result in there being a positive correlation between reproductive effort and levels of oxidative damage when such measurements are made several days later during peak reproduction. We would interpret such an association as evidence to suggest that reproduction has an oxidative cost. However, we are not aware of any evidence which links high levels of oxidative damage prior to breeding and high subsequent levels of offspring production. On the other hand, two studies have reported that levels of oxidative damage in females before, or early during reproduction, are significantly negatively correlated with the number of offspring that are subsequently produced (Costantini, Carello \& Fanfani, 2010; Stier et al., 2012). This suggests that pre-breeding oxidative state may constrain reproduction (Dowling \& Simmons, 2009; Stier et al., 2012).

Thirdly, it is possible that a positive correlation between reproductive effort and oxidative damage exists for breeders that had not experienced a manipulation of reproductive effort, as a consequence of 'individual quality' effects. For example, individuals that can afford high levels of reproductive effort may also be able to cope with high levels of oxidative damage, but this does not necessarily mean that increased reproductive effort causes increased oxidative damage. Similarly, the positive association between reproductive effort and oxidative damage could arise 
due to 'terminal investment', i.e. individuals in poorer physiological condition, with higher levels of oxidative damage and lower future survival prospects, may invest more in current reproduction. However, if this was true we may expect a non-linear association between reproduction and oxidative damage.

We conclude that since the positive correlation between reproductive effort and oxidative damage shown by our meta-analysis only holds for observational data, further experimental research will be needed to determine its causal basis. Nevertheless, whether or not reproduction causes oxidative damage, the idea that breeding females may be selected to reduce levels of oxidative damage in order to protect their developing offspring remains a valid hypothesis. Data from longitudinal sampling, and detailed cross-sectional sampling pre-, during and postreproduction will be critical for our understanding of the temporal dynamics of changes in oxidative damage and the potential costs. Indeed, only longitudinal data can reveal whether prebreeding oxidative state influences an individual's future reproductive effort (Stier et al., 2012). A capacity to meet the costs of oxidative shielding might influence an individuals' reproductive effort, or whether they breed at all. To explore these possibilities we need studies of how prebreeding oxidative state predicts subsequent reductions in oxidative damage associated with reproduction, and whether such changes correlate with variation in offspring number.

Finally, our meta-analysis considered only females; this reflects a bias in the published literature towards studies of females, presumably because it is often relatively difficult to measure reproductive effort in males (e.g. the cost of territory guarding and defence, and the cost of mate searching and guarding). Mitochondrial DNA appears to be particularly susceptible to oxidative damage, due to its proximity to the major source of ROS within cells (reviewed by Monaghan et al., 2009). On the assumption that reducing oxidative damage incurs energetic or 
other costs (discussed above; Section V, 4), it seems possible that females may pay a higher cost of reproduction than males due to oxidative shielding. If so, oxidative shielding might be a contributory factor underlying the evolution of inter-sexual conflict. However, several authors have emphasised the importance of oxidative damage as a mechanism responsible for decreased sperm viability and damage to male germline DNA; such changes in DNA could be transmitted to offspring via common genetic inheritance (mutations) and/or epigenetic inheritance, with deleterious consequences for the fitness of offspring. Females should be selected to avoid mating with such males (Blount, Møller \& Houston, 2001; Dowling \& Simmons, 2009; Metcalfe \& Alonso-Alvarez, 2010; Velando et al., 2008). Males, which we have not considered in our metaanalysis, might therefore gain from oxidative shielding of sperm to increase mating success or the viability of resulting offspring. Indeed, oxidative shielding could have an important role in preventing oxidative damage to germline DNA transmitted via the maternal or paternal line. Additional studies of temporal patterns of oxidative damage in reproducing and non-reproducing males would therefore be valuable.

\section{CONCLUSIONS}

(1) Our meta-analysis using data for females of homeothermic species reveals that, consistent with existing theory to explain the cost of reproduction, increased reproductive effort is associated with greater oxidative damage. This correlation holds for observational data, but not for data arising from experimental manipulations of reproductive effort. Therefore, further experimental research will be needed to determine its causal basis 
(2) Contrary to existing theory to explain the cost of reproduction, we found that transition to the reproductive state is associated with markedly reduced levels of oxidative damage in certain markers and tissues.

(3) We hypothesise that such reductions in oxidative damage are pre-emptive and function to shield mothers, and in particular their gametes and developing offspring, from critical levels of oxidative insults that inevitably increase as a consequence of reproductive effort. We refer to this idea as the 'oxidative shielding hypothesis'.

(4) The oxidative shielding hypothesis could explain why many existing studies have concluded that reproduction has little or no oxidative cost. Rather, according to our analysis, the cost of reproduction seems likely to be mediated by dual impacts of oxidative damage on mothers and offspring, while mothers may face additional costs diminishing such damage.

(5) We suggest that a valuable direction for future studies of the cost of reproduction would be to quantify the magnitude of the different costs associated with sustaining oxidative damage in mothers and their offspring, and those borne by mothers in the reduction of oxidative damage.

(6) It would also be interesting to extend the range of studies that quantify the oxidative cost of reproduction, to include additional data for males, and to examine whether breeding males exhibit 'oxidative shielding' and in which tissues. Similarly, it would be valuable to gather data for oxidative damage in organs of birds in relation to reproductive effort and reproductive state. (7) Current theory to explain the cost of reproduction focusses on the individual fitness costs faced by breeders. We suggest that future advance in life-history theory needs to take account of potential transgenerational impacts of the mechanisms underlying life-history trade-offs.

\section{ACKNOWLEDGEMENTS}


We are very grateful to C. Alonso-Alvarez, M. S. Benfato, P. Bergeron, D. Costantini, M.

Garratt, G. Markó, D. H. Nussey, C. Schmidt and Y.-C. Xu for providing further details on published analyses to enable their inclusion in the meta-analysis. We are also very grateful to $\mathrm{M}$.

J. P. Simons for discussion and advice regarding meta-analytical methods, and to N. B. Metcalfe, two anonymous reviewers, and the editors for many helpful suggestions which greatly improved the manuscript.

\section{REFERENCES}

Aksakal, E., Akaras, N., Tanboga, I. H., Kurt, M., Halici, Z., Odabasoglu, F. \& Unal, B. (2011). Relationship between oxidative stress and cardiomyopathic changes in ovariectomized rats. Cardiology 119, 235-241.

Albera, E. \& KANKOFER, M. (2009). Antioxidants in colostrum and milk of sows and cows. Reproduction in Domestic Animals 44, 606-611.

Al-Gubory, K. H., Fowler, P. A. \& GARREL, C. (2010). The roles of cellular reactive oxygen species, oxidative stress and antioxidants in pregnancy outcomes. International Journal of Biochemistry \& Cell Biology 42, 1634-1650.

Albera, E. \& Kankofer, M. (2009). Antioxidants in Colostrum and Milk of Sows and Cows. Reproduction in Domestic Animals 44, 606-611.

Alonso-Alvarez, C., Bertrand, S., Devevey, G., Prost, J., Faivre, B. \& Sorci, G. (2004). Increased susceptibility to oxidative stress as a proximate cost of reproduction. Ecology Letters 7, 363-368.

Alonso-Alvarez, C., Perez-Rodriguez, L., Garcia, J. T., Vinuela, J. \& Mateo, R. (2010). Age and breeding effort as sources of individual variability in oxidative stress markers in a bird species. Physiological and Biochemical Zoology 83, 110-118.

Beaulieu, M., Reichert, S., Le Maho, Y., Ancel, A. \& Criscuolo, F. (2011). Oxidative status and telomere length in a long-lived bird facing a costly reproductive event. Functional Ecology 25, 577-585.

Bergeron, P., Careau, V., Humphries, M. M., Reale, D., Speakman, J. R. \& Garant, D. (2011). The energetic and oxidative costs of reproduction in a free-ranging rodent. Functional Ecology 25, 1063-1071.

Bize, P., Devevey, G., Monaghan, P., Doligez, B. \& Christe, P. (2008). Fecundity and survival in relation to resistance to oxidative stress in a free-living bird. Ecology 89, 2584-2593.

Blanga-Kanfi, S., Miranda, H., Penn, O., Pupko, T., DeBry, R. W. \& Huchon, D. (2009). Rodent phylogeny revised: analysis of six nuclear genes from all major rodent clades. BMC Evolutionary Biology 9, 71.

Blount, J. D., Houston, D. C. \& Møller, A. P. (2000). Why egg yolk is yellow. Trends in Ecology \& Evolution 15, 47-49. 
Blount, J. D., Møller, A. P. \& Houston, D. C. (2001). Antioxidants, showy males and sperm quality. Ecology Letters 4, 393-396.

Borenstein, M., Hedges, L., Higgins, J. \& Rothstein, H. (2005). Comprehensive MetaAnalysis, Version 2. Biostat, Englewood, NJ. URL http://www.meta-analysis.com/.

Borenstein, M., Hedges, L. V., Higgins, J. P. T. \& Rothstein, H. R. (2010). A basic introduction to fixed-effect and random-effects models for meta-analysis. Research Synthesis Methods 1, 97-111.

Borras, C., Sastre, J., Garcia-Sala, D., Lloret, A., Pallardo, F. V. \& Vina, J. (2003). Mitochondria from females exhibit higher antioxidant gene expression and lower oxidative damage than males. Free Radical Biology and Medicine 34, 546-552.

Bose, P., Kadyrov, M., Goldin, R., Hahn, S., Backos, M., Regan, L. \& Huppertz, B. (2006). Aberrations of early trophoblast differentiation predispose to pregnancy failure: Lessons from the anti-phospholipid syndrome. Placenta 27, 869-875.

Bouwhuis, S., Charmantier, A., Verhulst, S. \& Sheldon, B. C. (2010). Trans-generational effects on ageing in a wild bird population. Journal of Evolutionary Biology 23, 636-642.

Bouwstra, R. J., Goselink, R. M. A., Dobbelaar, P., Nielen, M., Newbold, J. R. \& VAn WERVEN, T. (2008). The relationship between oxidative damage and vitamin $\mathrm{E}$ concentration in blood, milk, and liver tissue from vitamin E supplemented and nonsupplemented periparturient heifers. Journal of Dairy Science 91, 977-987.

BRAND, M. D. (2000). Uncoupling to survive? The role of mitochondrial inefficiency in ageing. Experimental Gerontology 35, 811-820.

Cartwright, S. J., Nicoll, M. A. C., Jones, C. G., Tatayah, V. \& Norris, K. (2014). Anthropogenic Natal Environmental Effects on Life Histories in a Wild Bird Population. Current Biology 24, 536-540.

Casagrande, S., Dell'Omo, G., Costantini, D., Tagliavini, J. \& Groothuis, T. (2011). Variation of a carotenoid-based trait in relation to oxidative stress and endocrine status during the breeding season in the Eurasian kestrel: A multi-factorial study. Comparative Biochemistry and Physiology a-Molecular \& Integrative Physiology 160, 16-26.

Castillo, C., Hernandez, J., Bravo, A., Lopez-Alonso, M., Pereira, V. \& Benedito, J. L. (2005). Oxidative status during late pregnancy and early lactation in dairy cows. Veterinary Journal 169, 286-292.

Cederberg, J., BAsU, S. \& ERIKSSON, U. J. (2001). Increased rate of lipid peroxidation and protein carbonylation in experimental diabetic pregnancy. Diabetologia 44, 766-774.

Chamberlain, S. A., Hovick, S. M., Dibble, C. J., Rasmussen, N. L., Van Allen, B. G., Maitner, B. S., Ahern, J. R., Bell-Dereske, L. P., Roy, C. L., MezA-Lopez, M., Carrillo, J., Siemann, E., Lajeunesse, M. J. \& Whitney, K. D. (2012). Does phylogeny matter? Assessing the impact of phylogenetic information in ecological metaanalysis. Ecology Letters 15, 627-636.

Chandra, G., Aggarwal, A., Singh, A. K., Kumar, M. \& Upadhyay, R. C. (2013). Effect of Vitamin E and Zinc Supplementation on Energy Metabolites, Lipid Peroxidation, and Milk Production in Peripartum Sahiwal Cows. Asian-Australasian Journal of Animal Sciences 26, 1569-1576.

CoHEN, J. (1988). Statistical power analysis for the behavioral sciences. Lawrence Erlbaum, Hillsdale, New Jersey.

Costantini, D. (2008). Oxidative stress in ecology and evolution: lessons from avian studies. Ecology Letters 11, 1238-1251. 
Costantini, D., CARello, L. \& FAnfani, A. (2010). Relationships among oxidative status, breeding conditions and life-history traits in free-living great tits Parus major and common starlings Sturnus vulgaris. Ibis 152, 793-802.

Costantini, D., Bonisoli-Alquati, A., Rubolini, D., CAPrioli, M., Ambrosini, R., Romano, M. \& SAINO, N. (2014a). Nestling rearing is antioxidant demanding in female barn swallows (Hirundo rustica). Naturwissenschaften 101, 541-8

Costantini D., Meillere A., Carravieri A., Lecomte V., Sorci G., Faivre B., Weimerskirch H., Bustamante P., Labadie P., H., B. \& O., C. (2014b). Oxidative stress in relation to reproduction, contaminants, gender and age in a long-lived seabird. Oecologia 175, 1107-16.

Costantini, D. \& Møller, A. P. (2008). Carotenoids are minor antioxidants for birds. Functional Ecology 22, 367-370.

Cram, D. L., Blount, J. D. \& Young, A. J. (2014). Oxidative stress and social dominance in a wild cooperative breeder. Functional Ecologypublished online ahead of print (doi: 10.1111/1365-2435.12317).

Da Silva, A. C. A., Salomon, T. B., Behling, C. S., Putti, J., Hackenhaar, F. S., Alabarse, P. V. G., Schuller, A. K. \& Benfato, M. S. (2013). Oxidative stress in the kidney of reproductive female rats during aging. Biogerontology 14, 411-422.

DAVIES, K. J. A. (2000). Oxidative stress, antioxidant defenses, and damage removal, repair, and replacement systems. Iubmb Life 50, 279-289.

Dowling, D. K. \& Simmons, L. W. (2009). Reactive oxygen species as universal constraints in life-history evolution. Proceedings of the Royal Society B-Biological Sciences 276, 1737 1745.

Fletcher, Q. E., Selman, C., Boutin, S., McAdam, A. G., Woods, S. B., Seo, A. Y., Leeuwenburgh, C., Speakman, J. R. \& Humphries, M. M. (2012). Oxidative damage increases with reproductive energy expenditure and is reduced by food-supplementation. Evolution 67, 1527-1536.

Garratt, M., McArdle, F., Stockley, P., Vasilaki, A., Beynon, R. J., Jackson, M. J. \& Hurst, J. L. (2012). Tissue-dependent changes in oxidative damage with male reproductive effort in house mice. Functional Ecology 26, 423-433.

Garratt, M., Pichaud, N., King, E. D. A. \& Brooks, R. C. (2013). Physiological adaptations to reproduction. I. Experimentally increasing litter size enhances aspects of antioxidant defence but does not cause oxidative damage in mice. Journal of Experimental Biology 216, 2879-2888.

Garratt, M., Vasilaki, A., Stockley, P., McArdle, F., Jackson, M. \& Hurst, J. L. (2011). Is oxidative stress a physiological cost of reproduction? An experimental test in house mice. Proceedings of the Royal Society B-Biological Sciences 278, 1098-1106.

Grune, T., Kramer, K., Hoppe, P. P. \& Siems, W. (2001). Enrichment of eggs with n-3 polyunsaturated fatty acids: Effects of vitamin E supplementation. Lipids 36, 833-838.

Gupta, S., Agarwal, A., Banerjee, J. \& Alvarez, J. G. (2007). The role of oxidative stress in spontaneous abortion and recurrent pregnancy loss: A systematic review. Obstetrical \& Gynecological Survey 62, 335-347.

HADFIELD, J. D. (2010). MCMC Methods for Multi-Response Generalized Linear Mixed Models: The MCMCglmm R Package. Journal of Statistical Software 33, 1-22.

Halliwell, B. \& GutTeridge, J. M. (1999). Free Radicals in Biology and Medicine. Oxford University Press., Oxford. 
Hammond, K. A., Konarzewski, M., Torres, R. M. \& Diamond, J. (1994). Metabolic ceilings under a combination of peak energy demands. Physiological Zoology 67, 1479-1506.

HAQ, A. U., BAILEY, C. A. \& CHINNAH, A. (1996). Effect of beta-carotene, canthaxanthin, lutein, and vitamin $\mathrm{E}$ on neonatal immunity of chicks when supplemented in the broiler breeder diets. Poultry Science 75, 1092-1097.

He, Z. Y., Yu, S. L., MeI, G., Zheng, M., Wang, M. L., Dai, Y. P., TANG, B. \& Li, N. (2008). Maternally transmitted milk containing recombinant human catalase provides protection against oxidation for mouse offspring during lactation. Free Radical Biology and Medicine 45, 1135-1142.

HEDGES, L. V. (1981). Distribution theory for Glass's estimator of effect size and related estimators. Journal of Educational and Behavioral Statistics 6, 107-128.

Hedges, S. B., MoberG, K. D. \& MAXson, L. R. (1990). Tetrapod phylogeny inferred from 18Sribosomal RNA sequences and a review of the evidence for amniote relationships. Molecular Biology and Evolution 7, 607-633.

HigGins, J. P. \& THOMPSON, S. G. (2002). Quantifying heterogeneity in a meta-analysis. Statistics in Medicine 21,1539-58.

Higgins, J. P., Thompson, S. G., Deeks, J. J. \& Altman, D. G. (2003). Measuring inconsistency in meta-analyses. British Medical Journal 327, 557-560.

Hozo, S. P., DJUlBEGOVIC, B., Hozo, I. (2005). Estimating the mean and variance from the median, range, and the size of a sample. BMC Medical Research Methodology 5, 13.

IsAKSSON, C., SHELDON, B. \& UlLER, T. (2011). The Challenges of Integrating Oxidative Stress into Life-history Biology. Bioscience 61, 194-202.

Jan, M. H., Das, G. K., Khan, F. A., Singh, J., Bashir, S. T., Khan, S., Prasad, J. K., Mehrotra, S., Pathak, M. C., Singh, G. \& SARKar, M. (2014). Evaluation of follicular oxidant-antioxidant balance and oxidative damage during reproductive acyclicity in water buffalo (Bubalus bubalis). Asian Pacific Journal of Reproduction 3, 35-40.

JaUniauX, E., POSTON, L. \& BURTON, G. J. (2006). Placental-related diseases of pregnancy: involvement of oxidative stress and implications in human evolution. Human Reproduction Update 12, 747-755.

King, E. D. A., GARRATt, M. \& BrooKs, R. (2013). Manipulating reproductive effort leads to changes in female reproductive scheduling but not oxidative stress. Ecology and Evolution 3, 4161-4171.

Kireev, R. A., Tresguerres, A. F., Vara, E., Ariznavarreta, C. \& Tresguerres, J. A. F. (2007). Effect of chronic treatments with $\mathrm{GH}$, melatonin, estrogens, and phytoestrogens on oxidative stress parameters in liver from aged female rats. Biogerontology 8, 469-482.

Koricheva, J., Gurevitch, J. \& Mengersen, K. (2014). Handbook of Meta-analysis in Ecology and Evolution. Princeton University Press.

Losdat, S., Helfenstein, F., Gaude, B. \& Richner, H. (2011). Reproductive effort transiently reduces antioxidant capacity in a wild bird. Behavioral Ecology 22, 1218-1226.

MARKó, G., Costantini, D., Michl, G. \& TOROK, J. (2011). Oxidative damage and plasma antioxidant capacity in relation to body size, age, male sexual traits and female reproductive performance in the collared Xycatcher (Ficedula albicollis). Journal of Comparative Physiology B-Biochemical Systemic and Environmental Physiology 181, 73-81. 
Metcalfe, N. B. \& Alonso-Alvarez, C. (2010). Oxidative stress as a life-history constraint: the role of reactive oxygen species in shaping phenotypes from conception to death. Functional Ecology 24, 984-996.

Metcalfe, N. B. \& Monaghan, P. (2013). Does reproduction cause oxidative stress? An open question. Trends in Ecology \& Evolution 28, 347-350.

Millon, A., Petty, S. J., Little, B. \& Lambin, X. (2011). Natal conditions alter age-specific reproduction but not survival or senescence in a long-lived bird of prey. Journal of Animal Ecology 80, 968-975.

Mohiti-Asli, M., Shariatmadari, F., Lotfollahian, H. \& Mazuji, M. T. (2008). Effects of supplementing layer hen diets with selenium and vitamin $\mathrm{E}$ on egg quality, lipid oxidation and fatty acid composition during storage. Canadian Journal of Animal Science 88, 475-483.

Monaghan, P., Metcalfe, N. B. \& Torres, R. (2009). Oxidative stress as a mediator of life history trade-offs: mechanisms, measurements and interpretation. Ecology Letters 12, 75 92.

Mourente, G., Tocher, D. R., Diaz, E., Grau, A. \& Pastor, E. (1999). Relationships between antioxidants, antioxidant enzyme activities and lipid peroxidation products during early development in Dentex dentex eggs and larvae. Aquaculture 179, 309-324.

MyATT, L. (2006). Placental adaptive responses and fetal programming. Journal of PhysiologyLondon 572, 25-30.

Noguera, J. C., Kim, S. Y. \& Velando, A. (2012). Pre-fledgling oxidative damage predicts recruitment in a long-lived bird. Biology Letters 8, 61-63.

Nussey, D. H., Kruuk, L. E. B., Morris, A. \& Clutton-Brock, T. H. (2007). Environmental conditions in early life influence ageing rates in a wild population of red deer. Current Biology 17, R1000-R1001.

Nussey, D. H., Pemberton, J. M., Pilkington, J. G. \& Blount, J. D. (2009). Life history correlates of oxidative damage in a free-living mammal population. Functional Ecology 23, 809-817.

Nystrom, T. (2005). Role of oxidative carbonylation in protein quality control and senescence. Embo Journal 24, 1311-1317.

Oldakowski, L., Piotrowska, Z., Chrzascik, K. M., Sadowska, E. T., Koteja, P. \& TAYLOR, J. R. E. (2012). Is reproduction costly? No increase of oxidative damage in breeding bank voles. Journal of Experimental Biology 215, 1799-1805.

Persky, A. M., Green, P. S., Stubley, L., Howell, C. O., Zaulyanov, L., Brazeau, G. A. \& SiMPKINS, J. W. (2000). Protective effect of estrogens against oxidative damage to heart and skeletal muscle in vivo and in vitro. Proceedings of the Society for Experimental Biology and Medicine 223, 59-66.

Pichaud, N., Garratt, M., Ballard, J. W. O. \& Brooks, R. C. (2013). Physiological adaptations to reproduction. II. Mitochondrial adjustments in livers of lactating mice. Journal of Experimental Biology 216, 2889-2895.

R DEVElopment CORE TEAM. (2014). R: A language and environment for statistical computing. R Foundation for Statistical Computing, Vienna, Austria. ISBN 3-900051-07-0, URL http://www.R-project.org/.

Rizzo, A., Ceci, E., Pantaleo, M., Mutinati, M., Spedicato, M., Minoia, G. \& Sciorsci, R. L. $(2013 a)$. Evaluation of blood and milk oxidative status during early postpartum of dairy cows. Animal 7, 118-123. 
Rizzo, A., Pantaleo, M., Mutinati, M., Minoia, G., Trisolini, C., Ceci, E. \& Sciorsci, R. L. $(2013 b)$. Blood and milk oxidative status after administration of different antioxidants during early postpartum in dairy cows. Research in Veterinary Science 95, 1171-1174.

Sainz, R. M., Reiter, R. J., MAyo, J. C., CABrera, J., TAN, D. X., QI, W. \& GARCiA, J. J. (2000). Changes in lipid peroxidation during pregnancy and after delivery in rats: effect of pinealectomy. Journal of Reproduction and Fertility 119, 143-149.

Schaff, C., Borner, S., Hacke, S., Kautzsch, U., Albrecht, D., Hammon, H. M., Rontgen, M. \& KuHLA, B. (2012). Increased anaplerosis, TCA cycling, and oxidative phosphorylation in the liver of dairy cows with intensive body fat mobilization during early lactation. Journal of Proteome Research 11, 5503-5514.

Schmidt, C. M., Blount, J. D. \& BennetT, N. C. (2014). Reproduction is associated with a tissue-dependent reduction of oxidative stress in eusocial female Damaraland mole-rats (Fukomys damarensis). PLoS One 9, e103286.

Selman, C., Blount, J. D., Nussey, D. H. \& Speakman, J. R. (2012). Oxidative damage, ageing, and life-history evolution: where now? Trends in Ecology \& Evolution 27, 570577.

Serini, S., Fasano, E., Piccioni, E., Cittadini, A. R. M. \& Calviello, G. (2011). Dietary n-3 Polyunsaturated fatty acids and the paradox of their health benefits and potential harmful effects. Chemical Research in Toxicology 24, 2093-2105.

Sibley, C. G. \& Ahlquist, J. E. (1990). Phylogeny and Classification of Birds: A Study in Molecular Evolution. Yale University Press, New Haven \& London.

Simons, M. J. P., COHEN, A. A. \& Verhulst, S. (2012). What does carotenoid-dependent coloration tell? Plasma carotenoid level signals immunocompetence and oxidative stress state in birds-a meta-analysis. Plos One 7, e43088.

Sinervo, B., Doughty, P., Huey, R. B. \& Zamudio, K. (1990) Allometric engineering: a causal analysis of natural selection on offspring size. Science 258, 1927-1930.

Song, S., LIU, L., EDWARDS, S. V. \& WU, S. Y. (2012). Resolving conflict in eutherian mammal phylogeny using phylogenomics and the multispecies coalescent model. Proceedings of the National Academy of Sciences of the United States of America 109, 14942-14947.

SPEAKMAN, J. R. (2008). The physiological costs of reproduction in small mammals. Philosophical Transactions of the Royal Society B-Biological Sciences 363, 375-398.

SPEAKMAN, J. R. \& GARRATT, M. (2014). Oxidative stress as a cost of reproduction: Beyond the simplistic trade-off model. Bioessays 36, 93-106.

StEARns, S. C. (1992). The Evolution of Life Histories. Oxford University Press, New York.

Sterne, J. A. C., Becker, B. J. \& Egger, M. (2005). The funnel plot. In Publication Bias in Meta-Analysis, p. 75-98. Ed. H. R. Rothstein, A. J. Sutton, and M. Borenstein, New York, John Wiley.

Stier, A., Reichert, S., Massemin, S., Bize, P. \& Criscuolo, F. (2012). Constraint and cost of oxidative stress on reproduction: correlative evidence in laboratory mice and review of the literature. Frontiers in Zoology 9, 37.

Suriyasathaporn, W., VinitKetKumnuen, U., CheWOnarin, T., ChuPia, V. \& PINYOPUMMINTR, T. (2009). The indicative influence of oxidative stress on low milk yields in dairy cattle. Thai Journal of Veterinary Medicine 39, 237-243.

TomruK, A., Guler, G. \& Dincel, A. S. (2010). The influence of $1800 \mathrm{MHz}$ GSM-like signals on hepatic oxidative DNA and lipid damage in nonpregnant, pregnant, and newly born rabbits. Cell Biochemistry and Biophysics 56, 39-47. 
Upreti, K., ChaKi, S. P. \& Misro, M. M. (2002). Evaluation of peroxidative stress and enzymatic antioxidant activity in liver and kidney during pregnancy and lactation in rats. Health and Population Perspectives and Issues 25, 177-185.

VAN DE CROMmenaCKer, J., Komdeur, J. \& Richardson, D. S. (2011). Assessing the cost of helping: the roles of body condition and oxidative balance in Seychelles warbler (Acrocephalus sechellensis). Plos One 6, e26423.

VAN NoORDWIJK, A. J. \& DE JONG, G. (1986). Acquisition and allocation of resources - their influence on variation in life history tactics. American Naturalist 128, 137-142.

Velando, A., Torres, R. \& Alonso-Alvarez, C. (2008). Avoiding bad genes: oxidatively damaged DNA in germ line and mate choice. Bioessays 30, 1212-1219.

VÉZINA, F. \& WiLliams, T.D. (2002). Metabolic costs of egg production in the European starling (Sturnus vulgaris). Physiological and Biochemical Zoology 75, 377-385.

Vial, C. A., Chen, Q., Liu, B. N., Hickey, A., Snowise, S., Salmon, J. E., Stone, P. R. \& ChAmley, L. W. (2013). Antiphospholipid antibodies internalised by human syncytiotrophoblast cause aberrant cell death and the release of necrotic trophoblast debris. Journal of Autoimmunity 47, 45-57.

Vina, J., Borras, C., Gambini, J., Sastre, J. \& Pallardo, F. V. (2005). Why females live longer than males? Importance of the upregulation of longevity-associated genes by oestrogenic compounds. Febs Letters 579, 2541-2545.

Wicheansoni, P., Hampanichrun, V., Chupia, V., Vinitchaikul, P. \& Suriyasathaporn, W. (2007). Effect of vitamin $E$ and selenium administration on concentration of malondialdehyde in udder milk. Journal of Animal Science 85, 9-10.

Wiersma, P., Selman, C., SPeAKman, J. R. \& Verhulst, S. (2004). Birds sacrifice oxidative protection for reproduction. Proceedings of the Royal Society of London Series BBiological Sciences 271, S360-S363.

WiLliams, G. C. (1966). Natural selection, the costs of reproduction, and a refinement Lack's principle. American Naturalist 100, 687-690.

Wilmut, I., SAles, D. I. \& AshwORTH, C. J. (1986). Maternal and embryonic factors associated with prenatal loss in mammals. Journal of Reproduction and Fertility 76, 851-864.

Xu, Y. C., YAng, D. B., Speakman, J. R. \& Wang, D. H. (2014). Oxidative stress in response to natural and experimentally elevated reproductive effort is tissue dependent. Functional Ecology 28, 402-410.

YANG, D. B., XU, Y. C., WANG, D. H. \& SpeAKMAn, J. R. (2013). Effects of reproduction on immuno-suppression and oxidative damage, and hence support or otherwise for their roles as mechanisms underpinning life history trade-offs, are tissue and assay dependent. Journal of Experimental Biology 216, 4242-4250.

\section{SUPPORTING INFORMATION}

Additional supporting information may be found in the online version of this article.

Table S1. Summary of sources of data used in the meta-analysis examining associations between reproductive effort and oxidative damage. 
Table S2. Summary of sources of data used in the meta-analysis examining associations between reproductive state (breeders compared with non-breeders) and oxidative damage. Appendix S1. Evaluating the importance of phylogeny in the meta-analysis.

Table S3. Evaluating the influence of phylogenetic relatedness on the relationship between reproduction and oxidative damage.

Fig. S1. Phylogenetic tree for association between reproductive effort and oxidative damage.

Fig. S2. Phylogenetic tree for categorical comparisons of breeders versus non-breeders.

Appendix S2. Evaluating potential sources of bias in the meta-analysis.

Fig. S3. Funnel plot for reproductive effort and oxidative damage ('all-tissues-all-markers').

Fig. S4. Funnel plot for reproductive state and liver protein carbonyls.

Fig. S5. Funnel plot for reproductive state and blood protein carbonyls.

Table S4. Results of the meta-analyses excluding subsets of data. 


\section{Figure legends}

Fig. 1. Relationship between reproductive effort and oxidative damage. For studies that measured multiple tissues and/or markers, the combined effect size was used for analysis; see Section III, 2-3, for details. Round symbols are effect sizes (Hedges' $g \pm 95 \%$ confidence intervals) based on continuous data or categorical comparison of relatively high versus lower effort for the relationship between offspring number and oxidative damage in mothers. Open symbols show effect sizes for the individual studies; the black symbol is the summary (i.e. overall) effect size. There was a significant positive association between reproductive effort and oxidative damage (random effects meta-analysis: $Z=2.714, N_{\text {studies }}=11, P=0.007$ ).

Fig. 2. Relationship between reproductive state and oxidative damage. Round symbols are effect sizes (Hedges' $g \pm 95 \%$ confidence intervals) based on the difference in mean oxidative damage of breeders and non-breeders. The black symbol denotes an analysis of all-tissues-all-markers; the grey shaded symbols denote analyses of subsets of data for individual-tissues-all-markers; and the open symbols denote analyses of subsets of data for individual-tissues-individualmarkers. Compared with non-breeders, negative values indicate lower oxidative damage in breeders, while positive values indicate higher oxidative damage in breeders. Markers are: MDA, malondialdehyde; OG, oxidised glutathione; PC, protein carbonyls; PT, protein thiols; ROM, reactive oxygen metabolites. The sign of the data for PT is reversed so negative values indicate decreased damage. Blood markers are plasma or serum. $Z$ values and two-tailed $P$-values arising from random-effects meta-analysis models are shown; statistically significant effect sizes are shown in bold type. $N_{\text {studies }}$ refers to the sample size of studies in each meta-analysis. See Section III, 2-3, for details. 
Fig. 3. Hypothesised schematic relationship between reproduction and oxidative damage, based on the results of the meta-analyses. The quantity $s$ ('shielding') is the Hedges' $g$ effect size for the difference in mean oxidative damage between non-breeders $\left(\bar{D}_{\mathrm{nb}}\right)$ and breeders $\left(\bar{D}_{\mathrm{b}}\right)$. From our analysis the value of $s$ is 0.828 (mean of point estimates for malondialdehyde in heart; protein carbonyls in heart, liver, and follicles; and protein thiols in liver; Fig. 2). Oxidative damage correlates positively with reproductive effort (based on continuous data and categorical comparison of relatively high versus lower effort; Fig. 1). We can represent the magnitude of this increase as Hedges' $g$ by dichotomising continuous data into 'high' $\left(R_{\text {high }}\right)$ and 'low' $\left(R_{\text {low }}\right)$ categories, above or below the mean reproductive effort of all breeders, $\bar{R}$, respectively. The magnitude of this effect (denoted $c$, 'cost of reproduction') is 0.343 . The range $R_{\text {low }}$ to $R_{\text {high }}$ is standardised as length 1; lines $s$ and $c$ are drawn in proportion to this unit length. 
Table 1. Heterogeneity analyses arising from the meta-analyses examining associations between reproduction and oxidative damage. Heterogeneity analyses were carried out where $N_{\text {studies }}>1$ (see Fig. 2). Markers are: MDA, malondialdehyde; PC, protein carbonyls; OG, oxidised glutathione; PT, protein thiols; ROM, reactive oxygen metabolites.

\begin{tabular}{|c|c|c|c|c|c|c|}
\hline \multirow[t]{2}{*}{ Comparison } & \multicolumn{2}{|c|}{ Level of analysis } & \multirow[t]{2}{*}{$Q$} & \multirow[t]{2}{*}{ d.f. $(Q)$} & \multirow[t]{2}{*}{$P$} & \multirow[t]{2}{*}{$I^{2}$} \\
\hline & Tissue & Marker & & & & \\
\hline Reproductive effort & All & All & 13.971 & 10 & 0.174 & 28.425 \\
\hline \multirow[t]{20}{*}{ Reproducing versus non-reproducing } & All & All & 90.435 & 17 & $<0.0001$ & 81.202 \\
\hline & Heart & All & 1.791 & 2 & 0.408 & 0.000 \\
\hline & & MDA & 0.125 & 1 & 0.724 & 0.000 \\
\hline & & $\mathrm{PC}$ & 0.116 & 1 & 0.734 & 0.000 \\
\hline & Liver & All & 79.338 & 8 & $<0.0001$ & 89.917 \\
\hline & & MDA & 103.159 & 7 & $<0.0001$ & 93.214 \\
\hline & & OG & 0.671 & 1 & 0.413 & 0.000 \\
\hline & & $\mathrm{PC}$ & 17.049 & 4 & 0.002 & 76.539 \\
\hline & & PT & 0.442 & 2 & 0.802 & 0.000 \\
\hline & Muscle & All & 0.221 & 3 & 0.974 & 0.000 \\
\hline & & MDA & 0.333 & 2 & 0.847 & 0.000 \\
\hline & & OG & 0.020 & 1 & 0.887 & 0.000 \\
\hline & & PT & 0.000 & 1 & 0.991 & 0.000 \\
\hline & Kidney & All & 19.220 & 3 & $<0.0001$ & 84.391 \\
\hline & & MDA & 31.363 & 3 & $<0.0001$ & 90.435 \\
\hline & & $\mathrm{PC}$ & 11.198 & 2 & 0.004 & 82.140 \\
\hline & Blood & All & 20.087 & 10 & 0.028 & 50.216 \\
\hline & & MDA & 11.027 & 7 & 0.137 & 36.522 \\
\hline & & ROM & 5.019 & 1 & 0.025 & 80.077 \\
\hline & & PC & 25.216 & 5 & $<0.0001$ & 80.171 \\
\hline
\end{tabular}

